\title{
Formation of electron traps in amorphous silica
}

\author{
Matteo Farnesi Camellone, ${ }^{1,2}$ Joachim C. Reiner, ${ }^{1}$ Urs Sennhauser, ${ }^{1}$ and Louis Schlapbach ${ }^{1,2}$ \\ ${ }^{1}$ EMPA, Swiss Federal Laboratories for Materials Testing and Research, Electronics/Metrology Laboratory, \\ 8600 Dübendorf, Switzerland \\ ${ }^{2}$ ETH, Physics Department, Swiss Federal Institute of Technology, CH-8093, Zürich, Switzerland \\ (Received 19 April 2007; revised manuscript received 9 July 2007; published 17 September 2007)
}

\begin{abstract}
The capture of an electron by a silicon atom in a defect-free bulk of amorphous silica $\left(a-\mathrm{SiO}_{2}\right)$ has been investigated. $A b$ initio constrained dynamics has been applied to evaluate the energy barrier. The computed energy barrier involved in the mechanism was found to be $0.23 \mathrm{eV}$. The electron trapping is accompanied by a strong local distortion of the tetrahedron of $\mathrm{Si}$ and $\mathrm{O}$ involved in the process. Hydrogen, which is an electronically active impurity of silicon dioxide, bonds to the oxygen of the Si-O silica bond on which the electron is localized. Upon restoring the neutrality of the system a paramagnetic configuration is found. The eigenvalues of the hyperfine tensor have been evaluated.
\end{abstract}

DOI: 10.1103/PhysRevB.76.125205

PACS number(s): 71.55.Jv, 72.20.Jv, 73.50.Gr

\section{INTRODUCTION}

The reliability of complementary metal-oxide semiconductor (CMOS) devices is a major concern in todays electronics. When these devices are scaled to smaller dimensions the gate oxide stops to work as an insulator and the rate of dielectric breakdown may be enhanced. Dielectric breakdown determines the lifetime of logical devices. The degradation of MOS structures has been related to the presence of atomistic defects in the gate oxide and at the interface between the silicon and the gate oxide. Another cause of degradation is the hydrogen released from the metal-oxide interface by hot electrons and diffusing into the semiconductor oxide. ${ }^{1,2}$ In particular, the class of oxygen vacancy defects, in the neutral or positively charged case, has been extensively studied both experimentally and theoretically. ${ }^{3}$ In a computer simulation, the oxygen vacancy is created artificially by removing one oxygen from the model system. The attempt to investigate defects in perfectly coordinated networks is instead much more demanding from a computational point of view because in most cases the underlying electronic structure theory, such as density functional theory (DFT), ${ }^{4}$ fails in describing such defects. ${ }^{5}$ The generation of traps during electrical stress involves physical changes in the oxide structure associated with bulk oxide bond breakage and rearrangement. Recently, Korkin et al. ${ }^{6}$ have proposed an alternative way to discuss the electron trap generation, where an electron can be trapped by an $\mathrm{Si}-\mathrm{O}$ bond weakening it and facilitating the bond breaking. In the calculation they used an $\mathrm{F}_{3} \mathrm{SiOSiF}_{3}$ molecule. They also postulated that the reaction with hydrogen can lead to a neutral $E^{\prime}$ center. The electron trap generation occurs when one takes into consideration that the injected electrons can interact with the localized gap states in the oxide. ${ }^{7}$

The aim of this work is to understand electron trap formation by adding an electron to a periodically repeated disordered and topologically defect-free network of silica $\left(a-\mathrm{SiO}_{2}\right)$. We investigated the model proposed by ${ }^{6}$ applying it to a periodically repeated $\mathrm{SiO}_{2}$ bulk. The energy barrier required for electron localization on an $\mathrm{Si}-\mathrm{O}$ bond has been explicitly evaluated by DFT calculations. Once the electron is localized the bond is weakened and can be broken more easily. Atomic hydrogen diffusing in the oxide bonds to the oxygen atom involved in the trapping mechanism and breaks the Si-O bond leading to a neutral $E^{\prime}$ center. The hyperfine values of this paramagnetic center have been evaluated and compared to other theories ${ }^{8}$ and experiments. ${ }^{9}$

\section{METHODS OF CALCULATIONS}

$A b$ initio structural relaxations have been performed within the framework of DFT and the Car-Parrinello molecular dynamics (CPMD) ${ }^{10}$ method. The electronic structure has been treated using plane waves (PWs) basis sets and pseudopotentials. We made use of the generalized gradient approximation $(\mathrm{GGA})^{11}$ on the exchange correlation energy. A plane wave basis set of $70 \mathrm{Ry}$ for plane waves and $280 \mathrm{Ry}$ for the electronic density has been used. Core-valence interactions have been described by a norm conserving Troullier-Martins pseudopotential $^{12}$ for oxygen and silicon atoms. The Brillouin zone has been sampled at the $\Gamma$ point. ${ }^{13} \mathrm{~A}$ fictitious mass of 700 a.u. for electrons and a time step of 0.12 fs have been used to integrate the equations of motion.

The periodic model of amorphous silica used in the simulations was generated by quenching from the melt using classical molecular dynamics (MD) with a final quenching rate of $1.6 \times 10^{12} \mathrm{~K} / \mathrm{s}$; the simulation cell is shown in Fig. 1 . During this preparation phase, the interaction between atoms has been modeled classically by using the empirical potential by van Beest, Kramer, and van Santen ${ }^{14}$ (BKS). The final configuration has been further relaxed using DFT. The model system contains 72 atoms ( 24 formula units) at the measured density of $2.20 \mathrm{~g} / \mathrm{cm}^{3}$. The chemical order is perfect with every silicon and oxygen atom being fourfold and twofold coordinated, respectively. The size of the periodically repeated supercell is $10.29 \times 10.29 \times 10.29 \AA^{3}$. The study of an amorphous system would require the use of larger samples to ensure that the distribution of structures is well-represented, in fact a glass can be in any one of the many possible network configurations that have nearly equal free energy. For this reason several model structures of 72 atoms of $a-\mathrm{SiO}_{2}$ were prepared and a detailed analysis of the structural prop- 


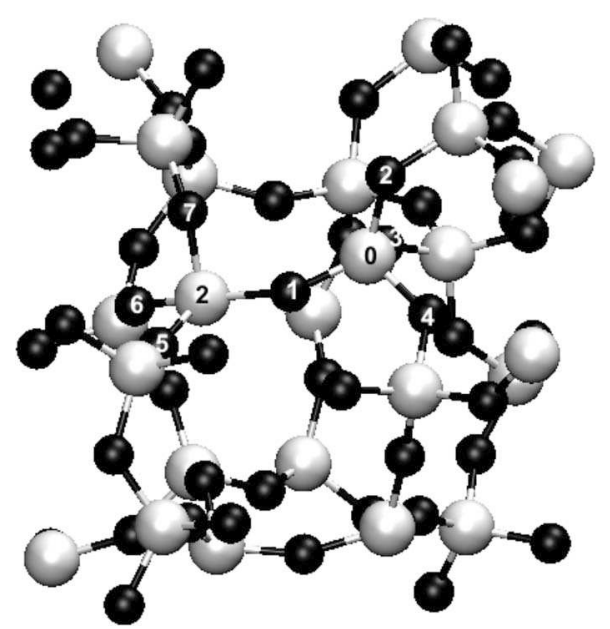

FIG. 1. Supercell of the periodic model of $a-\mathrm{SiO}_{2}$. The gray atoms are silicon and the black atoms oxygen. The atom labeled with 1 corresponds to the oxygen $\mathrm{O}(1)$ in the text, the atom labeled with 0 corresponds to the silicon $\operatorname{Si}(0)$.

erties of the generated systems has been carried out investigating properties such as bond lengths, angles, and ring statistics. Among those systems we selected one whose properties were in better agreement with structural experimental data.

\section{RESULTS}

\section{A. Neutral and negatively charged system}

The first goal of our study was to investigate the possibility of stable localized states in neutral and charged disordered systems. Ab initio constrained molecular dynamics have been applied to a disordered $\mathrm{SiO}_{2}$ network in its neutral state and in the presence of an extra electron added to the system.

The neutral spin singlet $a-\mathrm{SiO}_{2}$ was equilibrated for $\sim 1 \mathrm{ps}$ at $300 \mathrm{~K}$. We used constrained $a b$ initio molecular dynamics such that an $\mathrm{Si}-\mathrm{O}$ bond was progressively stretched (Fig. 2). Starting from the equilibrium configuration we evaluated the energy of a series of intermediate configurations in which all the degrees of freedom, except the $\mathrm{Si}(0)-\mathrm{O}(1)$ (see Fig. 1) distance (reaction coordinate), were relaxed. The initial equilibrium value of this bond was $1.64 \AA$ which is slightly larger than the experimental ${ }^{15}$ value (1.61 $\AA$ ), but can be explained with a general tendency of GGA. ${ }^{16}$ The structural relaxations have been performed using the CPMD annealing technique as implemented in the CPMD code. ${ }^{17}$ Each intermediate configuration has been relaxed for 7000 steps $(\sim 0.8 \mathrm{ps})$ in order to reach the $0 \mathrm{~K}$ configuration. The convergence criteria adopted for the optimization are $10^{-8}$ a.u. for the energy and $10^{-6}$ a.u. $\AA^{-1}$ for the forces. We found that the energy of the neutral system as a function of the $\mathrm{Si}(0)-\mathrm{O}(1)$ bond distance rapidly increases, see Fig. 2.

We subsequently generated a charged defect by adding one electron to the original $a-\mathrm{SiO}_{2}$ configuration. A uniform background charge with positive polarity has been added to

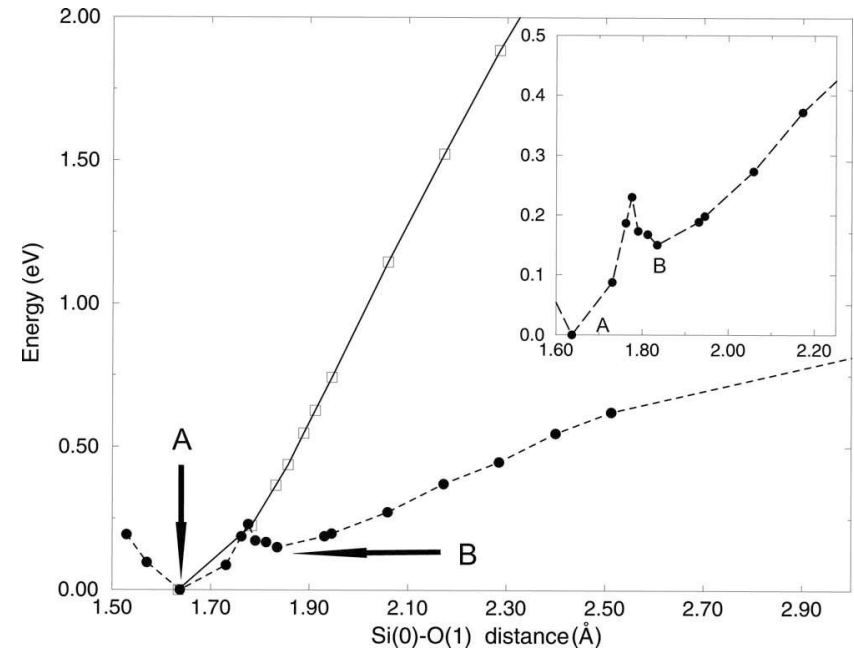

FIG. 2. Total energy of the neutral (solid line) and negatively charged system (dashed line) versus the $\mathrm{Si}(0)-\mathrm{O}(1)$ bond length. In the inset it is shown a magnification of the energy vs bond length in the negatively charged case. The labels $A$ and $B$ indicate the delocalized and the localized geometries, respectively.

restore the neutrality of the whole system and to prevent the unphysical divergence of the total energy arising from long range Coulomb interactions. ${ }^{18}$ In the calculations of the charged system the local spin density (LSD) approximation was used.

First of all, a structural relaxation of the negatively charged system has been performed. By computing the net spin density $\rho_{s}=\rho_{\uparrow}-\rho_{\downarrow}$ (where $\uparrow$ and means spin up and down, respectively) we noticed that adding one electron to the system leads to a configuration in which the net spin density was delocalized in space, as pointed out by Pacchioni et al. in Ref. 19. We will refer to this configuration as delocalized geometry, A in Fig. 2. We then identified a local minimum configuration in which the added electron localized on the $\mathrm{Si}(0)$ atom weakens the $\mathrm{Si}(0)-\mathrm{O}(1)$ bond; we will refer to this configuration as localized geometry, B in Fig. 2. We then imposed a constraint on the $\mathrm{Si}(0)-\mathrm{O}(1)$ bond and increased the reaction coordinate as in the neutral case. The energy of a series of intermediate configurations between $\mathrm{A}$ and B has been evaluated (see Fig. 2).

In the localized configuration the analysis of the spin distribution shows a high localization of the unpaired electron on the $\mathrm{Si}(0)$ atom. In addition, a shared electron number analysis has been performed on the $\mathrm{O}(1)-\mathrm{Si}(0)$ for the delocalized and localized geometry (see Table I). We computed a $0.23 \mathrm{eV}$ energy barrier separating the initial delocalized configuration from the final localized minimum energy configuration, with the localized configuration having a total energy which is $0.168 \mathrm{eV}$ higher than the delocalized configuration.

TABLE I. Shared electron number.

\begin{tabular}{lcc}
\hline \hline SEN & $\mathrm{Si}(2)-\mathrm{O}(1)$ & $\mathrm{Si}(0)-\mathrm{O}(1)$ \\
\hline Delocalized & 0.937 & 0.960 \\
Localized & 1.038 & 0.692 \\
\hline \hline
\end{tabular}


TABLE II. Mulliken charges analysis.

\begin{tabular}{|c|c|c|c|c|c|c|c|c|c|}
\hline $\begin{array}{l}q \\
\text { (a. u.) }\end{array}$ & $\operatorname{Si}(0)$ & $\mathrm{O}(2)$ & $\mathrm{O}(3)$ & $\mathrm{O}(4)$ & $\mathrm{O}(1)$ & $\operatorname{Si}(2)$ & $\mathrm{O}(5)$ & $\mathrm{O}(6)$ & $\mathrm{O}(7)$ \\
\hline Delocalized & 2.334 & -1.045 & -1.081 & -1.093 & -1.077 & 2.344 & -1.081 & -1.087 & -1.072 \\
\hline Localized & 1.753 & -1.029 & -1.068 & -1.073 & -1.002 & 2.313 & -1.090 & -1.090 & -1.071 \\
\hline
\end{tabular}

In the localized geometry the $\mathrm{Si}(0)-\mathrm{O}(1)$ bond reached the final value of $1.83 \AA$. In this minimum energy configuration the $\mathrm{O}(1)-\mathrm{Si}(0)$ and $\mathrm{Si}(0)-\mathrm{O}(2)$ bonds tend to align, which is leading to a significant distortion of the original tetrahedral structure. It results in a distorted trigonal pyramid with one axial and three equatorial neighbors, two of the equatorial bonds resulting longer than the axial and the third equatorial bonds. The $\mathrm{O}(1)-\mathrm{Si}(0)-\mathrm{O}(2)$ angle changes from the original value of $121.48^{\circ}$ to $156.44^{\circ}$. The added electron thus induces an $s p 2$-hybridization in the equatorial plane. To confirm that the obtained configuration was indeed an energy minimum, a vibrational analysis was performed. The frequencies obtained were real, thus signaling a minimum configuration (and not a saddle point) in the energy surface. The vibrational frequencies were deduced from the force constants, which in turn were evaluated from the Hellman-Feynman forces obtained by displacing all the silicon and oxygen atoms by $0.01 \AA$ away from their equilibrium positions for each direction of the Cartesian coordinates.

We then investigated the charge distribution in the system by computing the Mulliken charges (see Table II). Although the Mulliken analysis is subject to well-known ambiguities, ${ }^{20}$ we expect this approach to correctly reproduce trends.

As it can be inferred from Table II, there exists a significant difference of the charge distribution in the two configurations separated by the energy barrier. The Mulliken analysis of the delocalized geometry shows a symmetric distribution of the charges on the $\mathrm{Si}(0) \mathrm{O}_{i}(i=2,3,4)$ complex $(q=-0.903)$ and the $\mathrm{Si}(2) \mathrm{O}_{i}(i=5,6,7)(q=-0.896)$, while the localized geometry shows an asymmetric charge distribution with $\mathrm{Si}(0) \mathrm{O}_{i}(i=2,3,4) \quad(q=-1.417)$ and $\mathrm{Si}_{(2) \mathrm{O}_{i}(i}$ $=5,6,7)(q=-0.938)$ and the net spin density being localized on the $\mathrm{Si}(0)$ atom. The obtained configuration with the electron localized on the $\mathrm{Si}(0)$ atom was further relaxed including the self-interaction correction (SIC) scheme proposed in Ref. 21 (the same parameters proposed in Ref. 21 were used). This scheme represents a solution to the incomplete cancellation of the unpaired-electron self-interaction which contributes to the failure of DFT in describing self-trapping in general. ${ }^{5}$ The application of this functional did not lead to a significant change in the structure of the system, the $\mathrm{O}(1)-\mathrm{Si}(0)$ bond reached after relaxation the value of $1.95 \AA$ while the difference in energy $\Delta E$ between the configuration obtained with the SIC functional and that one obtained without the SIC functional was found to be $\Delta E=0.078 \mathrm{eV}$. To prove the generality of such a mechanism we applied the same procedure to another $\mathrm{Si}-\mathrm{O}$ bond. The same distortion was found with the localization of the extra electron on the $\mathrm{Si}$ atom. The $\mathrm{Si}-\mathrm{O}$ bond reached the value of $1.82 \AA$, after relaxation the involved angle reached a value of $157.24^{\circ}$. In this case the energy barrier has not been computed but the difference in energy between the two localized configurations was found to be $0.002 \mathrm{eV}$. Given the disordered nature of the amorphous network, a deeper analysis would require the investigation of more sites and different structures in order to get more statistics; we, however, think that this phenomenon can be responsible for rearrangement in the network.

Summarizing, ab initio constrained molecular dynamics has been applied to a disordered and topologically defectfree silica network; the energy of the whole system as a function of an $\mathrm{Si}-\mathrm{O}$ bond length has been evaluated. In the neutral case the energy progressively increases as a function of the bond length distance while in the presence of an extra electron an energy barrier of $0.23 \mathrm{eV}$ has been found. This barrier separates the starting delocalized minimum energy configuration from a local minimum energy configuration in which the extra electron localizes on the $\mathrm{Si}(0)$ silicon atom.

\section{B. Incorporation of atomic hydrogen and electron paramagnetic resonance (EPR) feature}

It is well-known that hydrogen is an electronically active impurity of silicon dioxide. The hydrogen is released from the metal-oxide interface upon collision with hot electrons. We investigated the behavior of the localized and the delocalized geometry in the presence of an atomic hydrogen. First an $\mathrm{H}^{0}$ was incorporated in the delocalized geometry of the $a-\mathrm{SiO}_{2}$ bulk (A in Fig. 2). After relaxation the $\mathrm{H}^{0}$ bonded to one of the $\mathrm{Si}$ atoms, resulting in a fivefold coordination with a bond length of about $1.52 \AA$. This result is in agreement with Ref. 22. Subsequently the same calculation was performed on the localized geometry (B in Fig. 2). Different starting positions for the added hydrogen have been investigated (eight in total). After relaxation we observed two different final configurations: one metastable in which the hydrogen bonded to the $\mathrm{O}(1)$ oxygen, with an $\mathrm{H}-\mathrm{O}(1)$ bond length of $1.06 \AA$. In this configuration the hydrogen pointed to the $\operatorname{Si}(0)$ atom and the distance between the $\operatorname{Si}(0)$ and $\mathrm{O}(1)$ atoms was $3.02 \AA$.In the other configuration the hydrogen bonded to an $\mathrm{Si}$ atom of the bulk resulting in delocalized configuration. The starting positions of the hydrogen which lead to the configuration in which the hydrogen bonded to the $\mathrm{O}(1)$ atom were found to be inside a sphere of radius $1.8 \AA$ centered on the $\mathrm{O}(1)$ atom. When placed outside this sphere, the hydrogen bonded to a neighbor $\mathrm{Si}$ atom. The energy of this configuration was found to be $0.4 \mathrm{eV}$ lower in energy with respect to the metastable one.

Then we investigated the behavior of the system in the presence of a proton $\mathrm{H}^{+}$, which makes the system again neutral. Also in this case different starting positions for the hydrogen atom have been chosen. Two different final configu- 


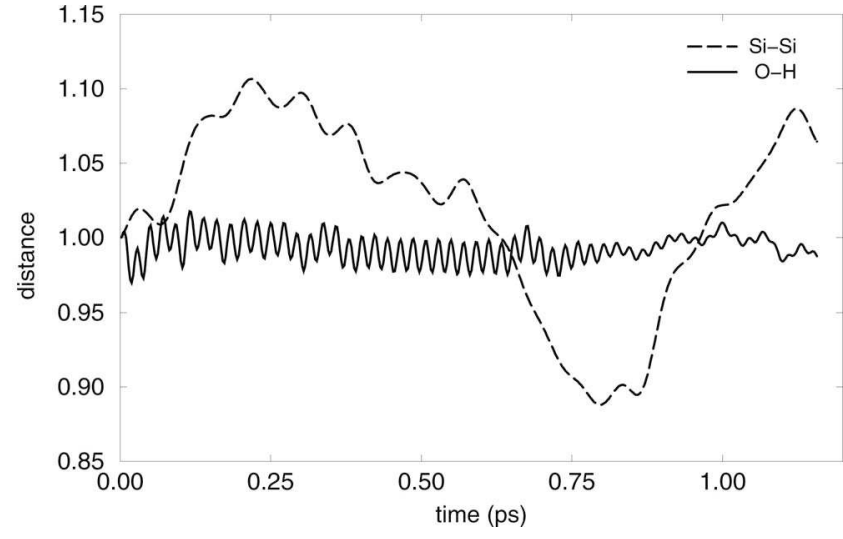

FIG. 3. O-H and $\mathrm{Si}(0)-\mathrm{Si}(2)$ normalized distance monitored during the $300 \mathrm{~K}$ simulation. The distance between the two atoms has been divided by the $0 \mathrm{~K}$ equilibrium distance.

rations were found: one in which the proton bonded to the $\mathrm{O}(1)$ atom with a bond length of $0.98 \AA$ and an $\mathrm{Si}(0)-\mathrm{Si}(2)$ distance of $3.23 \AA$, and one in which the hydrogen atom does not bond to any atoms in the network. As for the negative case we could identify a sphere of radius $1.8 \AA$ centered on the $\mathrm{O}(1)$ atom: all the starting positions of the hydrogen inside this region of the space lead to the final configuration in which the hydrogen bonded to the $\mathrm{O}(1)$ atom. The energy of this configuration was found to be $0.7 \mathrm{eV}$ lower than that of the interstitial hydrogen. In addition we studied the behavior of this configuration at room temperature. A microcanonical CPMD run at $300 \mathrm{~K}$ has been performed on the system monitoring the $\mathrm{O}-\mathrm{H}$ and $\mathrm{Si}(0)-\mathrm{Si}(2)$ distance over the trajectory (Fig. 3). The addition of the proton restored the neutrality of the system leading to a neutral $E^{\prime}$ center: ${ }^{23}$ a configuration with an unpaired electron on the $\operatorname{Si}(0)$. To make a connection with electron spin resonance (ESR) experiments the hyperfine values were calculated. The hyperfine Hamiltonian is expressed by $\hat{H}=\vec{S} \cdot \mathbf{A} \cdot \vec{I}$, where A is the hyperfine rank 2 tensor describing the coupling between the electronic spin $\vec{S}$ and the nuclear spin $\vec{I}$ at a given site $\mathbf{R}$. The components of the tensor $\mathbf{A}$ are $A_{i j}=a^{i s o} \delta_{i j}+a_{i j}^{a n i}$ where

$$
a^{i s o}=\frac{4 \pi}{3} \frac{g_{e} \mu_{e} g_{N} \mu_{N}}{\left\langle S_{Z}\right\rangle} \int d \mathbf{r} \rho_{s} \delta_{T}(\mathbf{r})
$$

and

$$
a_{i j}^{a n i}=\frac{1}{2} \frac{4 \pi}{3} \frac{g_{e} \mu_{e} g_{N} \mu_{N}}{\left\langle S_{Z}\right\rangle} \int d \mathbf{r} \rho_{s} \frac{3 r_{i} r_{j}-\delta_{i j} r^{2}}{r^{5}} .
$$

In the above expression $g_{e}$ is the free-electron $g$ factor, $\mu_{e}$ the Bohr magneton, $g_{N}$ the nuclear gyromagnetic ratio, and $\mu_{N}$ the nuclear magneton. The isotropic $a^{i s o}$ term corresponds to the Fermi contact interaction and the term $a_{i j}^{a n i}$ represents the dipole-dipole interaction. The hyperfine values have been computed within the Gaussian and augmented plane wave method theory using the code QUICKSTEP. ${ }^{24,25}$ The simulations have been performed using a triple-zeta valence polarization (TZVP) basis set, the Goedecker-TeterHutter pseudopotentials, and the local density approximation
TABLE III. Calculated mean eigenvalues.

\begin{tabular}{lccc}
\hline \hline $\begin{array}{l}A \\
(\mathrm{MHz})\end{array}$ & This work & Reference 8 & $\begin{array}{r}\text { Experiment } \\
\text { (Ref. 9) }\end{array}$ \\
\hline$A_{\text {strong }}$ & 1302.21 & 1442 & 1176 \\
$A_{\text {weak }}$ & 39.8 & 50 & 35 \\
\hline \hline
\end{tabular}

to the exchange correlation energy. The calculated mean eigenvalues of the weak and strong components of the hyperfine tensor of the neutral system are reported in Table III and compared to the experimental data of the $E^{\prime}$ center in positively charged $a-\mathrm{SiO}_{2}$ (Ref. 9) and to the results of Ref. 8 where another theory was used and the system was positively charged. The computed mean values compare well to the experimental results reported in Ref. 9.

Summarizing, we found that the addition of a $\mathrm{H}^{0}$ atom to the negatively charged system leads to two different configurations, one stable in which the $\mathrm{H}^{0}$ atom bonds to a silicon atom of the disordered network and one metastable in which the $\mathrm{H}^{0}$ atom bonds to the $\mathrm{O}(1)$ atom and whose energy is $0.4 \mathrm{eV}$ higher.

The addition of a $\mathrm{H}^{+}$atom to the system (restoring its neutrality) leads to two configurations, one in which the $\mathrm{H}^{+}$ does not bond to any atoms of the system and one in which the $\mathrm{H}^{+}$bonds to the $\mathrm{O}(1)$ atom leading to a neutral $E^{\prime}$ center, the energy of this configuration being $0.7 \mathrm{eV}$ lower. Finally the hyperfine values of the neutral $E^{\prime}$ center have been computed and compare well to experiments.

\section{Band structures and DOS}

The electronic structures (in the $\Gamma \rightarrow M$ direction) and the density of states (DOS) for the interstitial hydrogen, neutral defect, and negatively charged system have been computed

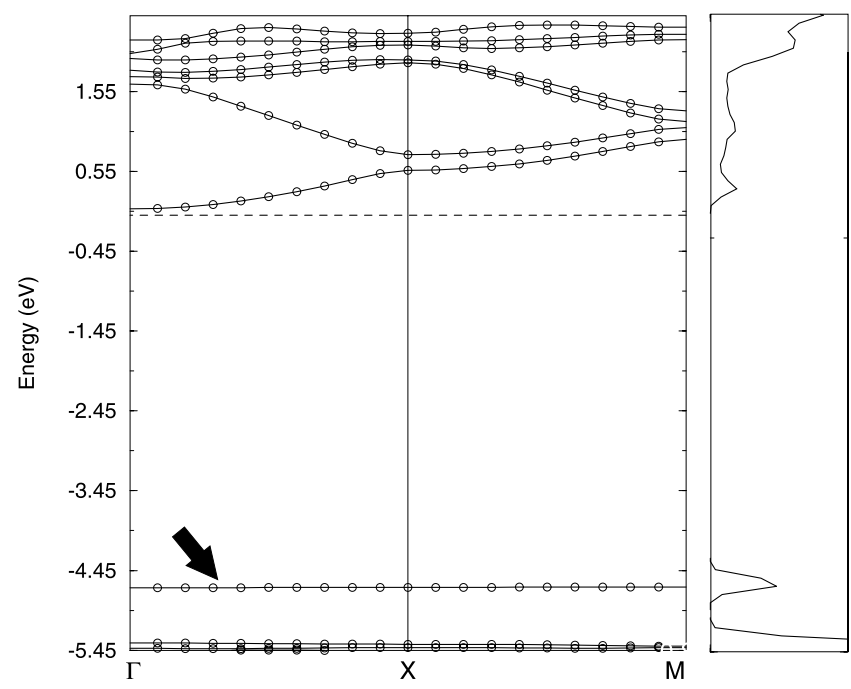

FIG. 4. Plot of the band structure (left) and density of states (right) for the interstitial hydrogen (see text) in the direction from $\Gamma$ to $M$. The arrow indicates the gap state induced by $\mathrm{H}$. The Fermi level has been set to $0 \mathrm{eV}$. 


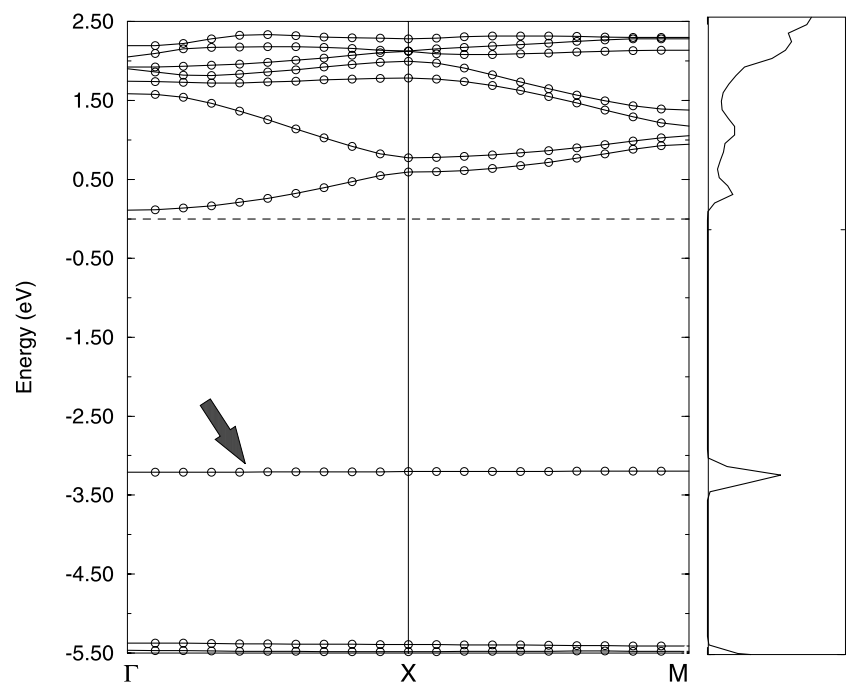

FIG. 5. Plot of the band structure (left) and density of states (right) for the localized geometry (see text) in the direction from $\Gamma$ to $M$. The arrow indicates the gap state induced by $\mathrm{H}$. The Fermi level has been set to $0 \mathrm{eV}$.

by using the Quantum-ESPRESSO package. ${ }^{26}$ Calculations have been performed by using a $3 \times 3 \times 3 \quad k$-points Monkhorst-Pack mesh ${ }^{27}$ in the integration of the Brillouin zone (BZ), this corresponds to $42 k$-points. In Fig. 4 the calculated electronic structure for the interstitial hydrogen configuration is shown. The hydrogen induced gap state (indicated by an arrow) differs from Ref. 22, where a similar calculation for $\alpha$-quartz has been carried out. The electronic structure corresponds to a superposition of those of $a-\mathrm{SiO}_{2}$ and the $\mathrm{H}$ atom. In Fig. 5 the calculated electronic structure of the neutral defect is shown. In this case the gap state induced by $\mathrm{H}$ (indicated by an arrow) shifts toward the middle of the band gap. Figure 6 shows a plot of the computed electronic structure of the configuration in which an extra electron has been added. The electron originated gap can be discussed in terms of $n$ doping. When the impurity electron is added a level appears in the gap while the Fermi level must adjust itself to preserve the neutrality shifting towards the conduction band. ${ }^{28}$

\section{CONCLUSIONS}

In summary, we have calculated the energy barrier and the density of states for an electron trapped in a chemically ordered and periodically repeated $a-\mathrm{SiO}_{2}$ network; a stable lo-

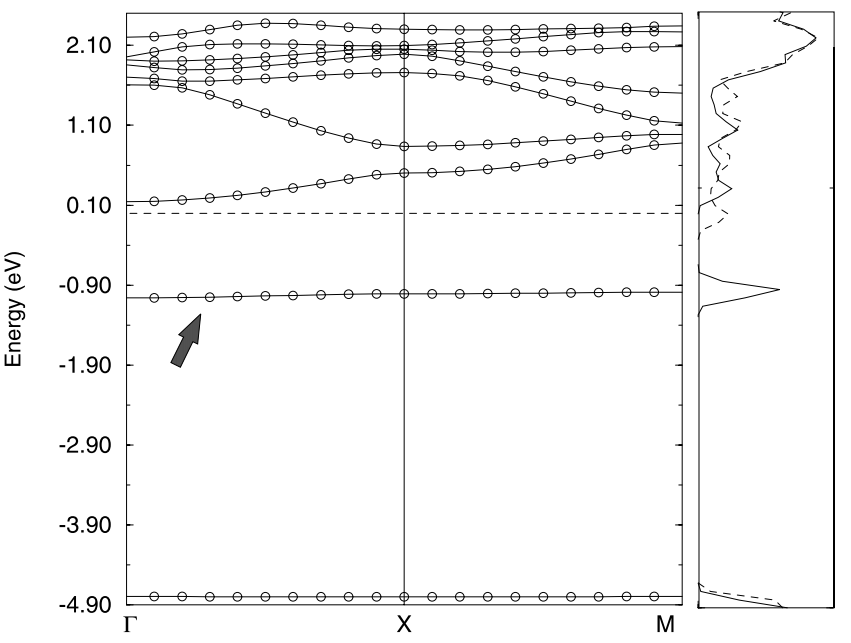

FIG. 6. Plot of the band structure (left) and ( $\alpha$ solid line and $\beta$ dashed line) density of states (right) for the localized geometry (see text) in the direction from $\Gamma$ to $M$. The arrow indicates the gap state induced by the added electron. The Fermi level has been set to $0 \mathrm{eV}$.

cal minimum in which the added electron localizes on a $\mathrm{Si}$ atom, weakening the $\mathrm{Si}-\mathrm{O}$ bond, was found. The behavior of the system in the presence of a $\mathrm{H}^{0}$ or $\mathrm{H}^{+}$atom has been investigated. Different configurations have been found according to the starting position of the hydrogen atom. If hydrogen is placed within a sphere or radius $1.8 \AA$ centered on the $\mathrm{O}(1)$ atom it always bonds to that oxygen atom. When a proton attaches to the oxygen it weakens the bond giving rise to a neutral $E^{\prime}$ center configuration. The ESR hyperfine parameters of the neutral configuration were evaluated, and a remarkable agreement to experiment was found. Finally the band structures and density of states of the interstitial hydrogen, proton incorporation, and negatively charged system have been computed.

Additional calculations, experiments, and statistics would help to understand better and deeper the nature and mechanism of such a defect induced by the hosted electron.

\section{ACKNOWLEDGMENTS}

We thank J. Hutter and Ari P. Seitsonen for insightful discussions. One of the authors (M. F.) would like to thank Daniele Passerone and Alfredo Pasquarello for their very constructive suggestions, ideas, and careful reading of the paper. All the calculations have been performed at CSCS.
${ }^{1}$ D. L. Griscom, J. Electron. Mater. 21, 762 (1992).

${ }^{2}$ D. J. DiMaria, E. Cartier, and D. Arnold, J. Appl. Phys. 73, 3367 (1993)

${ }^{3}$ A. Stirling and A. Pasquarello, Phys. Rev. B 66, 245201 (2002).

${ }^{4}$ P. Hohenberg and W. Kohn, Phys. Rev. 136, B864 (1964); W. Kohn and L. J. Sham, ibid. 140, A1133 (1965).
${ }^{5}$ G. Pacchioni, F. Frigoli, D. Ricci, and J. A. Weil, Phys. Rev. B 63, 054102 (2001).

${ }^{6}$ G. Bersuker, A. Korkin, Y. Jeon, and H. R. Huff, Appl. Phys. Lett. 80, 832 (2001).

${ }^{7}$ G. Bersuker, Y. Jeon, and H. R. Huff, Microelectron. Reliab. 41, 1923 (2001). 
${ }^{8}$ M. Boero, A. Pasquarello, J. Sarnthein, and R. Car, Phys. Rev. Lett. 78, 887 (1997).

${ }^{9}$ D. L. Griscom, Phys. Rev. B 22, 4192 (1980).

${ }^{10}$ R. Car and M. Parrinello, Phys. Rev. Lett. 55, 2471 (1985).

${ }^{11}$ J. P. Perdew, J. A. Chevary, S. H. Vosko, K. A. Jackson, M. R. Pederson, D. J. Singh, and C. Fiolhais, Phys. Rev. B 46, 6671 (1992).

${ }^{12}$ N. Troullier and J. L. Martins, Phys. Rev. B 43, 1993 (1991).

${ }^{13}$ G. Galli and A. Pasquarello, Computar Simulation in Chemical Physics, edited by M. P. Allen and D. J. Tildesley (Kluwer Academic Publishers, Dordrecht, Netherlands, 1993), pp. 261-313.

${ }^{14}$ B. W. H. van Beest, G. J. Kramer, and R. A. van Santen, Phys. Rev. Lett. 64, 1955 (1990).

${ }^{15}$ P. A. V. Johnson, A. C. Wright, and R. N. Sinclar, J. Non-Cryst. Solids 58, 109 (1983).

${ }^{16}$ A. Dal Corso, A. Pasquarello, A. Baldereschi, and R. Car, Phys. Rev. B 53, 1180 (1996).

${ }^{17}$ http://www.cpmd.orgCPMD code by J. Hutter et al., (Max-PlanckInsitut FKF and IBM Zurich Research Laboratory, 1995-2005).
${ }^{18}$ D. Marx and J. Hutter, in Modern Methods and Algorithms of Quantum Chemistry, Forshungszentrum Jülich, NIC Series, Vol. 1, 2nd ed. edited by J. Grotendorst(John von Neumann Institut for Computing, Jülich. 2000), pp. 301-449.

${ }^{19}$ G. Pacchioni and C. Mazzeo, Phys. Rev. B 62, 5452 (2000).

${ }^{20}$ R. S. Mulliken, J. Chem. Phys. 23, 1833 (1955).

${ }^{21}$ J. VandeVondele and M. Sprik, Phys. Chem. Chem. Phys. 7, 1363 (2005).

${ }^{22}$ A. Yokozawa and Y. Miyamoto, Phys. Rev. B 55, 13783 (1997).

${ }^{23}$ P. M. Lenahan et al., J. Non-Cryst. Solids 266, 835 (2000).

${ }^{24}$ http://cp2k.berlios.de/

${ }^{25}$ R. Declerck, E. Pauwels, V. Van Speybroeck, and M. Waroquier, Phys. Rev. B 74, 245103 (2006).

${ }^{26} \mathrm{http}$ //quantum-espresso.org

${ }^{27}$ H. J. Monkhorst and J. D. Pack, Phys. Rev. B 13, 5188 (1976).

${ }^{28}$ S. M. Sze, Physics of Semiconductor Devices, 2nd ed. (Wiley, New York, 1969). 